\title{
Nonuniform biorthogonal wavelets on positive half line via Walsh Fourier transform
}

\author{
Owais Ahmad ${ }^{1 *} \mathbb{D}$, Neyaz A. Sheikh ${ }^{1}$ and Mobin Ahmad²
}

\section{${ }^{*}$ Correspondence:}

siawoahmad@gmail.com

${ }^{1}$ Department

of Mathematics, National

Institute of Technology,

Srinagar, Jammu and Kashmir

190006, India

Full list of author information

is available at the end of the

article

\begin{abstract}
In this article, we introduce the notion of nonuniform biorthogonal wavelets on positive half line. We first establish the characterizations for the translates of a single function to form the Riesz bases for their closed linear span. We provide the complete characterization for the biorthogonality of the translates of scaling functions of two nonuniform multiresolution analysis and the associated biorthogonal wavelet families in $L^{2}\left(\mathbb{R}^{+}\right)$. Furthermore, under the mild assumptions on the scaling functions and the corresponding wavelets associated with nonuniform multiresolution analysis, we show that the wavelets can generate Reisz bases.
\end{abstract}

Keywords: Nonuniform biorthogonal wavelet, Nonuniform multiresolution analysis, Walsh-Fourier transform

Mathematics Subject Classification: 42C40, 42C15, 43A70, 11585

\section{Introduction}

The theory of wavelet transforms have emanated as a broadly used tool in various disciplines of science and engineering including image processing, spectrometry, turbulence, computer graphics, optics and electromagnetism, telecommunications, DNA sequence analysis, quantum physics, solution of differential equations. In context of signal processing, it has been assumed that orthogonality is the key property for synthesis and analysing signals. In order to study a higher-level signal processing, biorthogonality plays a vital role in which two sets are incorporated: one serves for the analysis and the other one for synthesis. Towars the culminating years of 1990's, biorthogonal wavelets are considered as cornerstone technique in image compression due to their natural feature of concentrating energy in a few transform coefficients and advantageous over orthogonal wavelets, by relaxing orthonormal to biorthogonal, additional degrees of freedom are added to design problems. Biorthogonal wavelets in $L^{2}(\mathbb{R})$ were investigated by Bownik and Garrigos [1], Cohen et al. [2], Chui and Wang [3]. The numerical aspect of biorthogonal wavelets were studied by Karoui and Vaillancourt [4].

Multiresolution analysis is the heart of wavelet analysis as it gives a general framework for analysing wavelet systems. All the signals in real life applications are not obtained from the uniform shifts. For the analysis and decomposition of these signals by means of stable mathematical technique, Gabardo and Nashed [5] introduced a notion of nonuniform author(s) and the source, provide a link to the Creative Commons licence, and indicate if changes were made. The images or other third party material in this article are included in the article's Creative Commons licence, unless indicated otherwise in a credit line to the material. If material is not included in the article's Creative Commons licence and your intended use is not permitted by statutory regulation or exceeds the permitted use, you will need to obtain permission directly from the copyright holder. To view a copy of this licence, visit http:// creativecommons.org/licenses/by/4.0/. 
MRA where the translation set acting on the scaling function associated with the MRA to generate the subspace $\mathcal{V}_{0}$ is no longer a group, but is the union of $\mathbb{Z}$ and a translate of $\mathbb{Z}$. Shah and Abdullah [6] established NUMRA on non-Archimedean local fields.

In the recent years, the development of wavelet theory in the context of Walsh analysis have been extensively studied by many authors including Farkov [7], Meenakshi [8] but still more concepts need to be studied for its enhancement. Recently, Ahmad and his collaborators investigated wavelet frames [9], nonuniform wavelet frames [10-14], Nonuniform $p$-tight wavelet frames [15], Tight Framelets [16, 17], wavepacket systems [18, 19], Frames associated with shift invariant spaces [20], Gabor frames [21], numerical study of wavelets [22-30] and obtained many interested results. Continuing our research on wavelet and wavelet frames, we in this paper introduce the notion of nonuniform biorthogonal wavelets in $L^{2}\left(\mathbb{R}^{+}\right)$. We obtain the characterization for the translates of a single function to form the Riesz bases for their closed linear span. We also provide a complete characterization for the biorthogonality of the translates of scaling functions of two NUMRA's and the associated biorthogonal wavelet families. Moreover, under mild assumptions on the scaling functions and the corresponding wavelets, we show that the nonuniform wavelets can generate Reisz bases for $L^{2}\left(\mathbb{R}^{+}\right)$.

The article is structured as follows. In Section "Methods", we recall methods of Fourier analysis on positive half line including basic definitions of MRA and NUMRA . In Section "Results and discussion", we establish necessary and sufficient conditions for the translates of a function to form a Riesz basis for its closed linear span. Furthermore, we show that the wavelets associated with dual MRA's are biorthogonal and generate Riesz bases for $L^{2}\left(\mathbb{R}^{+}\right)$.

\section{Methods}

Let $\mathbb{R}^{+}, \mathbb{Z}^{+}$and $\mathbb{N}$ respectively denotes the set of nonnegative real numbers, set of non-negative integers and the set of natural numbers. By the symbols $[x]$ and $\{x\}$, we mean the integer and fractional part of $x$ respectively. Let $p>1$ be a fixed natural number. For $\xi \in \mathbb{R}^{+}$ and any integer $j>0$, we set

$$
\xi_{j}=\left[p^{j} \xi\right](\bmod p), \quad \xi_{-j}=\left[p^{1-j} \xi\right](\bmod p),
$$

where $\xi_{j}, \xi_{-j} \in\{0,1, \ldots, p-1\}$. Clearly, $\xi_{j}$ and $\xi_{-j}$ are the digits in the $p$-ary expansion of $\xi$ :

$$
\xi=\sum_{j<0} \xi_{-j} p^{-j-1}+\sum_{j>0} \xi_{j} p^{-j}
$$

The first sum on the right is always finite and

$$
[\xi]=\sum_{j<0} \xi_{-j} p^{-j-1}, \quad\{\xi\}=\sum_{j>0} x_{j} p^{-j}
$$

On $\mathbb{R}^{+}$the addition is defined in the following manner:

$$
\xi \oplus \eta=\sum_{j<0} \zeta_{j} p^{-j-1}+\sum_{j>0} \zeta_{j} p^{-j}
$$


with $\zeta_{j}=\xi_{j}+\eta_{j}(\bmod p), j \in \mathbb{Z} \backslash\{0\}$, where $\zeta_{j} \in\{0,1, \ldots, p-1\}$ and $\xi_{j}, \eta_{j}$ are given by (2.1). Clearly, $[\xi \oplus \eta]=[\xi] \oplus[\eta]$ and $\{\xi \oplus \eta\}=\{\xi\} \oplus\{\eta\}$. We write $\delta=\xi \ominus \eta$ if $\delta \oplus \eta=\xi$, where $\ominus$ denotes subtraction modulo $p$ on $\mathbb{R}^{+}$.

Let $\varepsilon_{p}=\exp (2 \pi i / p)$, we define a function $s_{0}(x)$ on $[0,1)$ by

$$
s_{0}(\xi)= \begin{cases}1, & \text { if } \xi \in[0,1 / p) \\ \varepsilon_{p}^{\ell}, & \text { if } \xi \in\left[\ell p^{-1},(\ell+1) p^{-1}\right), \quad \ell \in\{1,2, \ldots, p-1\} .\end{cases}
$$

The system of generalized Walsh functions $\left\{w_{m}(\xi): m \in \mathbb{Z}^{+}\right\}$on $[0,1)$ is defined in the following way:

$$
w_{0}(\xi) \equiv 1 \quad \text { and } \quad w_{m}(\xi)=\prod_{j=0}^{k}\left(s_{0}\left(p^{j} x\right)\right)^{\mu_{j}}
$$

where $m=\sum_{j=0}^{k} \mu_{j} p^{j}, \mu_{j} \in\{0,1, \ldots, p-1\}, \mu_{k} \neq 0$. These functions form a complete orthogonal system. A finite linear combination of Walsh functions is known as Walsh polynomial. For $\xi, \eta \in \mathbb{R}^{+}$, let

$$
\chi(\xi, \eta)=\exp \left(\frac{2 \pi i}{p} \sum_{j=1}^{\infty}\left(\xi_{j} \eta_{-j}+\xi_{-j} \eta_{j}\right)\right),
$$

where $\xi_{j}, \eta_{j}$ are defined as in (2.1).

It is easy to see that

$$
\chi\left(\xi, \frac{m}{p^{n}}\right)=\chi\left(\frac{\xi}{p^{n}}, m\right)=w_{m}\left(\frac{\xi}{p^{n}}\right), \quad \forall \xi \in\left[0, p^{n}\right), m, n \in \mathbb{Z}_{+},
$$

and

$$
\chi(\xi \oplus \eta, \delta)=\chi(\xi, \delta) \chi(\eta, \delta), \quad \chi(\xi \ominus \eta, \delta)=\chi(\xi, \delta) \overline{\chi(\eta, \delta)},
$$

where $\xi, \eta, \delta \in \mathbb{R}_{+}$and $\xi \oplus \eta$ is $p$-adic irrational. It is well known that systems $\{\chi(\alpha, .)\}_{\alpha=0}^{\infty}$ and $\{\chi(\cdot, \alpha)\}_{\alpha=0}^{\infty}$ form an orthonormal bases in $L^{2}[0,1]$ (See [31, 32]).

For a function $\phi \in L^{1}\left(\mathbb{R}^{+}\right) \cap L^{2}\left(\mathbb{R}^{+}\right)$, the Walsh-Fourier transform is defined as

$$
\widehat{\phi}(\zeta)=\int_{\mathbb{R}_{+}} \phi(\xi) \overline{\chi(\xi, \zeta)} \mathrm{d} \xi
$$

where $\chi(\xi, \zeta)$ is given by (2.2). The Walsh-Fourier operator $\mathfrak{F}: L^{1}\left(\mathbb{R}^{+}\right) \cap L^{2}\left(\mathbb{R}^{+}\right) \rightarrow L^{2}\left(\mathbb{R}^{+}\right), \mathfrak{F} f=\widehat{f}$, extends uniquely to the whole space $L^{2}\left(\mathbb{R}^{+}\right)$. The Walsh-Fourier transform enjoys similar properties to those of the classic Fourier transform [31-34]. In particular, if $\phi \in L^{2}\left(\mathbb{R}^{+}\right)$, then $\widehat{\phi} \in L^{2}\left(\mathbb{R}^{+}\right)$and

$$
\|\widehat{\phi}\|_{L^{2}\left(\mathbb{R}^{+}\right)}=\|\phi\|_{L^{2}\left(\mathbb{R}^{+}\right)} .
$$

Furthermore, if $\phi \in L^{2}[0,1]$, then we can define the Walsh-Fourier coefficients of $\phi$ as 


$$
\widehat{\phi}(n)=\int_{0}^{1} \phi(\xi) \overline{w_{n}(\xi)} d x .
$$

The series $\sum_{n \in \mathbb{Z}_{+}} \widehat{\phi}(n) w_{n}(\xi)$ is called the Walsh-Fourier series of $\phi$. From the standard $L^{2}$ -theory, we can observe that the Walsh-Fourier series of $\phi$ converges to $\phi$ in $L^{2}[0,1]$ and Parseval's identity holds:

$$
\|\phi\|_{2}^{2}=\int_{0}^{1}|\phi(\xi)|^{2} \mathrm{~d} \xi=\sum_{\mathrm{n} \in \mathbb{Z}_{+}}|\widehat{\phi}(\mathrm{n})|^{2} .
$$

By $p$-adic interval $I \subset \mathbb{R}_{+}$of range $n$, we mean intervals of the form

$$
I=I_{n}^{\ell}=\left[\ell p^{-n},(\ell+1) p^{-n}\right), \quad \ell \in \mathbb{Z}_{+} .
$$

Each of these $p$-adic intervals is both closed and open under the $p$-adic topology which is generated by the collection of $p$-adic intervals [31]. The collection $\left\{\left[0, p^{-j}\right): j \in \mathbb{Z}\right\}$ forms a fundamental system of the $p$-adic topology on $\mathbb{R}^{+}$. Therefore, the generalized Walsh functions $w_{j}(\xi), 0 \leq j \leq p^{n}-1$, assume constant values on each of $p$-adic interval $I_{n}^{\ell}$ and hence continuous on these intervals. Thus, $w_{j}(\xi)=1$ for $\xi \in I_{n}^{0}$.

Let $\mathcal{E}_{n}\left(\mathbb{R}^{+}\right)$denotes the space of $p$-adic entire functions of order $n$. Thus, for every $\phi \in \mathcal{E}_{n}\left(\mathbb{R}^{+}\right)$, we have

$$
\phi(\xi)=\sum_{k \in \mathbb{Z}^{+}} \phi\left(p^{-n} k\right) \chi_{I_{n}^{k}}(\xi), \quad \xi \in \mathbb{R}_{+} .
$$

It is clear that $\mathcal{E}_{n}\left(\mathbb{R}^{+}\right)$contains each Walsh function of order up to $p^{n-1}$. The set $\mathcal{E}\left(\mathbb{R}^{+}\right)$ of $p$-adic entire functions on $\mathbb{R}^{+}$is the union of all the spaces $\mathcal{E}_{n}\left(\mathbb{R}^{+}\right)$and is dense in $L^{p}\left(\mathbb{R}^{+}\right), 1 \leq p<\infty$ and each function in $\mathcal{E}\left(\mathbb{R}^{+}\right)$is of compact support. Thus, we consider the following set of functions

$$
\mathcal{E}^{0}\left(\mathbb{R}^{+}\right)=\left\{\phi \in \mathcal{E}\left(\mathbb{R}^{+}\right): \widehat{\phi} \in L^{\infty}\left(\mathbb{R}^{+}\right) \text {and supp } \phi \subset \mathbb{R}^{+} \backslash\{0\}\right\}
$$

Let $N \geq 1$ be a given integer and $r$ be an odd integer which are relatively prime such that $1 \leq r \leq N-1$, we consider the translation set $\Lambda^{+}$as

$$
\Lambda^{+}=\left\{0, \frac{r}{N}\right\}+\mathbb{Z}^{+} .
$$

It can be easily seen that the translation set $\Lambda^{+}$is not necessarily a group nor a uniform discrete set. The set $\Lambda^{+} \mathrm{n}$ is the union of $\mathbb{Z}$ and a translate of $\mathbb{Z}$. Furthermore, the translation set $\Lambda^{+}$is the spectrum for the spectral set $\Gamma_{N}=\left[0, \frac{1}{2}\right) \cup\left[\frac{N}{2}, \frac{N+1}{2}\right)$ and the pair $\left(\Lambda^{+}, \Gamma_{N}\right)$ is called a spectral pair [5].

Definition 2.1 Let $N \geq 1$ be a given integer and $r$ be an odd integer which are relatively prime such that $1 \leq r \leq N-1$, an associated nonuniform MRA is a sequence of closed subspaces $\left\{\mathcal{V}_{j}: j \in \mathbb{Z}\right\}$ of $L^{2}\left(\mathbb{R}^{+}\right)$satisfying the following properties:

(a) $\mathcal{V}_{j} \subset \mathcal{V}_{j+1} \forall j \in \mathbb{Z}$; 
(b) $\bigcup_{j \in \mathbb{Z}} \mathcal{V}_{j}$ is dense in $L^{2}\left(\mathbb{R}^{+}\right)$;

(c) $\bigcap_{j \in \mathbb{Z}} \mathcal{V}_{j}=\{0\}$;

(d) $g(x) \in \mathcal{V}_{j} \Longleftrightarrow g(N x) \in \mathcal{V}_{j+1} \forall j \in \mathbb{Z}$;

(e) There exists a function $\phi \in \mathcal{V}_{0}$ such that $\left\{\phi(x \ominus \sigma): \sigma \in \Lambda^{+}\right\}$, is a complete orthonormal basis for $\mathcal{V}_{0}$.

It should be noted that the definition of dyadic dilation multiresolution analysis in one dimension can be deduced from the above definitio when $N=1$. For $N>1$, the dilation factor of $N$ corroborates that $N \Lambda^{+} \subset \mathbb{Z}^{+} \subset \Lambda^{+}$.

For every $j \in \mathbb{Z}$, define $\mathcal{W}_{j}$ as the orthogonal complement of $\mathcal{V}_{j}$ in $\mathcal{V}_{j+1}$. Thus we can write

$$
\mathcal{V}_{j+1}=\mathcal{V}_{j} \oplus \mathcal{W}_{j} \quad \text { and } \quad \mathcal{W}_{m} \perp \mathcal{W}_{m^{\prime}} \quad \text { if } m \neq m^{\prime} .
$$

Therefore, it implies that for $j>M$,

$$
\mathcal{V}_{j}=\mathcal{V}_{M} \oplus \bigoplus_{m=0}^{j-M-1} \mathcal{W}_{j-m}
$$

By invoking Definition 2.2. (b), this follows that

$$
L^{2}(\mathbb{R})=\bigoplus_{j \in \mathbb{Z}} \mathcal{W}_{j}
$$

a decomposition of $L^{2}\left(\mathbb{R}^{+}\right)$into mutually orthogonal subspaces.

There exists $N-1$ functions whose translated and dilated family form an orthonormal basis for $L^{2}\left(\mathbb{R}^{+}\right)$.

Definition 2.3 A set $\left\{\psi_{\ell}: 1 \leq \ell \leq N-1\right\} \subset L^{2}(\mathbb{R})$ is said to be a set of basic wavelets associated with the nonuniform multiresolution analysis $\left\{\mathcal{V}_{j}: j \in \mathbb{Z}\right\}$ if the family of functions $\left\{\psi_{\ell}(x \ominus \sigma): 1 \leq \ell \leq N-1, \sigma \in \Lambda^{+}\right\}$forms an orthonormal basis for $\mathcal{W}_{0}$.

\section{Results and Discussion}

Lemma 3.1 Let $\phi, \widetilde{\phi} \in L^{2}\left(\mathbb{R}^{+}\right)$be given. Then the collection $\left\{\phi(x \ominus \sigma): \sigma \in \Lambda^{+}\right\}$is biorthogonal to $\left\{\widetilde{\phi}(x \ominus \sigma): \sigma \in \Lambda^{+}\right\}$if and only if

$$
\sum_{\sigma \in \Lambda_{+}^{+}} \widehat{\phi}(\zeta \oplus \sigma) \overline{\overline{\widetilde{\phi}}(\zeta \oplus \sigma)}=1 \quad \text { a.e } \zeta \in \mathbb{R}^{+} .
$$

Proof For $\gamma \in \Lambda^{+}$, it follows that $\langle\phi(x \ominus \sigma), \widetilde{\phi}(x \ominus \gamma)\rangle=\delta_{\sigma, \gamma} \Leftrightarrow\langle\phi, \widetilde{\phi}(x \ominus \gamma)\rangle=\delta_{0, \gamma}$. Moreover, we have 


$$
\begin{aligned}
\langle\phi, \widetilde{\phi}(x \ominus \gamma)\rangle & =\langle\widehat{\phi}, \widehat{\widetilde{\phi}}(x \ominus \gamma)\rangle \\
& =\int_{\mathbb{R}^{+}} \widehat{\phi}(\zeta) \overline{\widetilde{\phi}}(\zeta) \overline{\chi(\gamma, \zeta)} \mathrm{d} \zeta \\
& =\int_{0}^{1 / 2}\left\{\sum_{m \in \mathbb{Z}} \widehat{\phi}\left(\zeta \oplus \frac{m}{2}\right) \overline{\widetilde{\hat{\phi}}\left(\zeta \oplus \frac{m}{2}\right)} \chi(\gamma, m)\right\} \overline{\chi(\gamma, \zeta)} \mathrm{d} \zeta .
\end{aligned}
$$

Using the fact that $\left\{\overline{\chi(\gamma, \zeta)}: \gamma \in \Lambda^{+}\right\}$is an orthonormal basis of $L^{2}\left[0, \frac{1}{2}\right)$, we obtain the desired result.

Now we proceed to establish a sufficient condition for the translates of a function to be linearly independent.

Lemma 3.2 Let $\phi \in L^{2}\left(\mathbb{R}^{+}\right)$. Suppose there exists two constants $C, D>0$ such that

$$
C \leq \sum_{\sigma \in \Lambda^{+}}|\widehat{\phi}(\zeta \oplus \sigma)|^{2} \leq D \quad \text { for a.e } \zeta \in \mathbb{R}^{+} .
$$

Then, the set $\left\{\phi(x \ominus \sigma): \sigma \in \Lambda^{+}\right\}$is linearly independent.

Proof For the proof of the lemma, it is sufficient to find another function say $\widetilde{\phi}$ whose translates are biorthogonal to $\phi$. To do this, we define the function $\widetilde{\phi}$ by

$$
\widehat{\widetilde{\phi}}(\zeta)=\frac{\widehat{\phi}(\zeta)}{\sum_{\sigma \in \Lambda^{+}}|\widehat{\phi}(\zeta \oplus \sigma)|^{2}} .
$$

Equation (3.1) implies that $\widetilde{\phi}$ is well defined and

$$
\begin{aligned}
\sum_{\gamma \in \Lambda^{+}} \widehat{\phi}(\zeta \oplus \gamma) \overline{\widetilde{\phi}}(\zeta \oplus \gamma) & =\sum_{\gamma \in \Lambda^{+}} \widehat{\phi}(\zeta \oplus \gamma) \frac{\overline{\widehat{\phi}(\zeta \oplus \gamma)}}{\sum_{\sigma \in \Lambda^{+}}|\widehat{\phi}(\zeta \oplus \sigma \oplus \gamma)|^{2}} \\
& =\frac{\sum_{\gamma \in \Lambda^{+}}|\widehat{\phi}(\zeta \oplus \gamma)|^{2}}{\sum_{\nu \in \sigma}|\widehat{\phi}(\zeta+\nu)|^{2}} \\
& =1 .
\end{aligned}
$$

Applying Lemma 3.1, it follows that the set $\left\{\phi(x \ominus \sigma): \sigma \in \Lambda^{+}\right\}$is linearly independent. Thus the proof is completed.

Lemma 3.3 Assume that the scaling function $\phi$ satisfies inequality (3.1). Let $g=\sum_{\sigma \in \Lambda^{+}} h_{\sigma} \phi(x \ominus \sigma)$, where $g \in \operatorname{span}\left\{\phi(x \ominus \sigma): \sigma \in \Lambda^{+}\right\}$and $\left\{h_{\sigma}\right\}$ is a finite sequence. Define the Fourier transform of $h$ by $\widehat{h}(\zeta)=\sum_{\sigma \in \Lambda^{+}} h_{\sigma} \overline{\chi(\sigma, \zeta)}$. Then 


$$
C \int_{0}^{1 / 2}|\widehat{h}(\zeta)|^{2} \mathrm{~d} \zeta \leq\|\mathrm{g}\|_{2}^{2} \leq \mathrm{D} \int_{0}^{1 / 2}|\widehat{\mathrm{h}}(\zeta)|^{2} \mathrm{~d} \zeta
$$

Proof By using Placherel's theorem, we obtain

$$
\begin{aligned}
\int_{\mathbb{R}^{+}}|g(x)|^{2} d x & =\int_{\mathbb{R}^{+}}\left|\sum_{\sigma \in \Lambda^{+}} h_{\sigma} \phi(x \ominus \sigma)\right|^{2} d x \\
& =\int_{\mathbb{R}^{+}}\left|\sum_{\sigma \in \Lambda^{+}} h_{\sigma} \widehat{\phi}(\zeta) \overline{\chi(\sigma, \zeta)}\right|^{2} \mathrm{~d} \zeta \\
& =\int_{\mathbb{R}^{+}}|\widehat{\phi}(\zeta)|^{2}\left|\sum_{\sigma \in \Lambda^{+}} h_{\sigma} \overline{\chi(\sigma, \zeta)}\right|^{2} \mathrm{~d} \zeta \\
& =\int_{\mathbb{R}^{+}}|\widehat{\phi}(\zeta)|^{2}|\widehat{h}(\zeta)|^{2} \mathrm{~d} \zeta \\
& =\int_{0}^{1 / 2} \sum_{m \in \mathbb{Z}}\left|\widehat{\phi}\left(\zeta \oplus \frac{m}{2}\right)\right|^{2}|\widehat{h}(\zeta)|^{2} \mathrm{~d} \zeta .
\end{aligned}
$$

Using identity (3.1), the result follows.

Theorem 3.4 Let $\left\{\phi(x \ominus \sigma): \sigma \in \Lambda^{+}\right\}$be a Riesz basis for its closed linear span. Suppose that there exists a function $\left\{\widetilde{\phi}(x \ominus \sigma): \sigma \in \Lambda^{+}\right\}$which is biorthogonal to $\left\{\phi(x \ominus \sigma): \sigma \in \Lambda^{+}\right\}$. Then, for every $f \in \overline{\operatorname{span}}\left\{\phi(x \ominus \sigma): \sigma \in \Lambda^{+}\right\}$, we have

$$
f=\sum_{\sigma \in \Lambda^{+}}\langle f, \widetilde{\phi}(x \ominus \sigma)\rangle \phi(x \ominus \sigma)
$$

and there exists constants $C, D>0$ such that

$$
C\|f\|_{2}^{2} \leq \sum_{\sigma \in \Lambda^{+}}|\langle f, \widehat{\widetilde{\phi}}(\zeta \ominus \sigma)\rangle|^{2} \leq D\|f\|_{2}^{2} .
$$

Proof We first prove (3.2) and (3.3) for any $f \in \operatorname{span}\left\{\phi(x \ominus \sigma): \sigma \in \Lambda^{+}\right\}$and then generalize it to $\overline{\operatorname{span}}\left\{\phi(x \ominus \sigma): \sigma \in \Lambda^{+}\right\}$. Let $f \in \operatorname{span}\left\{\phi(x \ominus \sigma): \sigma \in \Lambda^{+}\right\}$, then there exists a finite sequence $\left\{h_{\sigma}: \sigma \in \Lambda^{+}\right\}$such that $f=\sum_{\sigma \in \Lambda^{+}} h_{\sigma} \phi(x \ominus \sigma)$. Also, the biorthogonality condition implies that

$$
\begin{aligned}
\langle f, \widetilde{\phi}(x \ominus \gamma)\rangle & =\left\langle\sum_{\sigma \in \Lambda^{+}} h_{\sigma} \phi(x \ominus \sigma), \widetilde{\phi}(x \ominus \gamma)\right\rangle \\
& =\sum_{\sigma \in \Lambda^{+}} h_{\sigma}\langle\phi(x \ominus \sigma), \widetilde{\phi}(x \ominus \gamma)\rangle \\
& =h_{\sigma},
\end{aligned}
$$

which proves (3.2). In order to prove (3.3), we make the use of Lemma 3.3 to get 


$$
D^{-1}\|f\|_{2}^{2} \leq \int_{0}^{1 / 2}|\widehat{h}(\zeta)|^{2} \mathrm{~d} \zeta \leq \mathrm{C}^{-1}\|\mathrm{f}\|_{2}^{2}
$$

Using the Placherel formula for Fourier series and the fact that $h_{\sigma}=\langle f, \widetilde{\phi}(x \ominus \sigma)\rangle$, we obtain

$$
\int_{0}^{1 / 2}|\widehat{h}(\zeta)|^{2} \mathrm{~d} \zeta=\sum_{\sigma \in \Lambda^{+}}\left|\mathrm{h}_{\sigma}\right|^{2}=\sum_{\sigma \in \Lambda^{+}}|\langle\mathrm{f}, \widetilde{\phi}(\mathrm{x} \ominus \sigma)\rangle|^{2} .
$$

This proves (3.3). We now generalize the results to $\overline{\operatorname{span}}\left\{\phi(x \ominus \sigma): \sigma \in \Lambda^{+}\right\}$. For $f \in \overline{\operatorname{span}}\left\{\widetilde{\phi}(x \ominus \sigma): \sigma \in \Lambda^{+}\right\}$, there exists a sequence $\left\{f_{m}: m \in \mathbb{Z}\right\}$ in $\operatorname{span}\left\{\widetilde{\phi}(x \ominus \sigma): \sigma \in \Lambda^{+}\right\}$such that $\left\|f_{m}-f\right\|_{2} \rightarrow 0$ as $m \rightarrow \infty$. Thus, for each $\sigma \in \Lambda^{+}$, we have

$$
\left\langle f_{m}, \widetilde{\phi}(x \ominus \sigma)\right\rangle \rightarrow\langle f, \widetilde{\phi}(x \ominus \sigma)\rangle \quad \text { as } m \rightarrow \infty .
$$

Hence, the result holds for each $f_{m}$. Thus, we have

$$
\begin{aligned}
\sum_{\sigma \in \Lambda^{+}}|\langle f, \widetilde{\phi}(x \ominus \sigma)\rangle|^{2} & =\sum_{\sigma \in \Lambda^{+}} \lim _{m \rightarrow \infty}\left|\left\langle f_{m}, \widetilde{\phi}(x \ominus \sigma)\right\rangle\right|^{2} \\
& =\lim _{m \rightarrow \infty} \sum_{\sigma \in \Lambda^{+}}\left|\left\langle f_{m}, \widetilde{\phi}(x \ominus \sigma)\right\rangle\right|^{2} \\
& \leq D \lim _{m \rightarrow \infty}\left\|f_{m}\right\|_{2}^{2} \\
& =D\|f\|_{2}^{2} .
\end{aligned}
$$

Moreover, we have

$$
\left\{\sum_{\sigma \in \Lambda^{+}}\left|\left\langle f_{m}, \widetilde{\phi}(x \ominus \sigma)\right\rangle\right|^{2}\right\}^{1 / 2} \leq\left\{\sum_{\sigma \in \Lambda^{+}}\left|\left\langle f_{m}-f, \widetilde{\phi}(x \ominus \sigma)\right\rangle\right|^{2}\right\}^{1 / 2}+\left\{\sum_{\sigma \in \Lambda^{+}}|\langle f, \widetilde{\phi}(x \ominus \sigma)\rangle|^{2}\right\}^{1 / 2} .
$$

As the upper bound in (3.3) holds for $f_{m}-f$ and lower bound for each $f_{m}$, we infer that

$$
C^{1 / 2}\|f\|_{2} \leq D^{1 / 2}\left\|f_{m}-f\right\|_{2}+\left(\sum_{\sigma \in \Lambda^{+}}\left|\left\langle f_{m}, \widetilde{\phi}(x \ominus \sigma)\right\rangle\right|^{2}\right)^{1 / 2}
$$

from which we conclude that

$$
C\|f\|_{2}^{2} \leq \sum_{\sigma \in \Lambda^{+}}|\langle f, \widetilde{\phi}(x \ominus \sigma)\rangle|^{2}
$$

Combining (3.4) and (3.5), we obtain (3.3). Similarly, we can prove (3.2) for 


$$
f \in \overline{\operatorname{span}}\left\{\phi(x \ominus \sigma): \sigma \in \Lambda^{+}\right\}
$$

and the proof is completed.

Now we proceed to establish the properties of Nonuniform Biorthogonal wavelets on positive half line.

Let $\left\{\mathcal{V}_{j}: j \in \mathbb{Z}\right\}$ and $\left\{\widetilde{\mathcal{V}}_{j}: j \in \mathbb{Z}\right\}$ be biorthogonal NUMRA's with scaling functions $\phi$ and $\widetilde{\phi}$. Then there exists integral periodic functions $m_{0}$ and $\widetilde{m}_{0}$ with the property $\widehat{\phi}(\zeta)=m_{0}(\zeta / N) \widehat{\phi}(\zeta / N)$ and $\widehat{\widetilde{\phi}}(\zeta)=\widetilde{m}_{0}(\zeta / N) \widehat{\widetilde{\phi}}(\zeta / N)$. Suppose there exists integral periodic functions $m_{\ell}$ and $\widetilde{m}_{\ell}, 1 \leq \ell \leq N-1$ such that

$$
\mathcal{M}(\zeta) \overline{\widetilde{\mathcal{M}}}(\zeta)=I
$$

where

$$
\mathcal{M}(\zeta)=\left(\begin{array}{cccc}
m_{0}\left(\frac{\zeta}{N}\right) & m_{0}\left(\frac{\zeta}{N} \oplus \frac{1}{2 N}\right) & \ldots & m_{0}\left(\frac{\zeta}{N} \oplus \frac{N-1}{2 N}\right) \\
m_{1}\left(\frac{\zeta}{N}\right) & m_{2}\left(\frac{\zeta}{N} \oplus \frac{1}{2 N}\right) & \ldots & m_{2}\left(\frac{\zeta}{N} \oplus \frac{N-1}{2 N}\right) \\
\vdots & \vdots & \ddots & \vdots \\
m_{N-1}\left(\frac{\zeta}{N}\right) & m_{N-1}\left(\frac{\zeta}{N} \oplus \frac{1}{2 N}\right) & \ldots & m_{N-1}\left(\frac{\zeta}{N} \oplus \frac{N-1}{2 N}\right)
\end{array}\right)
$$

and

$$
\widetilde{\mathcal{M}}(\zeta)=\left(\begin{array}{cccc}
\widetilde{m}_{0}\left(\frac{\zeta}{N}\right) & \widetilde{m}_{0}\left(\frac{\zeta}{N} \oplus \frac{1}{2 N}\right) & \ldots & \widetilde{m}_{0}\left(\frac{\zeta}{N} \oplus \frac{N-1}{2 N}\right) \\
\widetilde{m}_{1}\left(\frac{\zeta}{N}\right) & \widetilde{m}_{2}\left(\frac{\zeta}{N} \oplus \frac{1}{2 N}\right) & \ldots & \widetilde{m}_{2}\left(\frac{\zeta}{N} \oplus \frac{N-1}{2 N}\right) \\
\vdots & \vdots & \ddots & \vdots \\
\widetilde{m}_{N-1}\left(\frac{\zeta}{N}\right) & \widetilde{m}_{N-1}\left(\frac{\zeta}{N} \oplus \frac{1}{2 N}\right) & \ldots & \widetilde{m}_{N-1}\left(\frac{\zeta}{N} \oplus \frac{N-1}{2 N}\right)
\end{array}\right) .
$$

For $1 \leq \ell \leq N-1$, define the associated biorthgonal wavelets as $\psi_{\ell}$ and $\widetilde{\psi}_{\ell}$ by

$$
\widehat{\psi}_{\ell}(\zeta)=m_{\ell}(\zeta / N) \widehat{\phi}(\zeta / N) \quad \text { and } \quad \widehat{\widetilde{\psi}}_{\ell}(\zeta)=\widetilde{m}_{\ell}(\zeta / N) \widehat{\widetilde{\phi}}(\zeta / N)
$$

Definition 3.5 A pair of NUMRA's $\left\{\mathcal{V}_{j}: j \in \mathbb{Z}\right\}$ and $\left\{\widetilde{\mathcal{V}}_{j}: j \in \mathbb{Z}\right\}$ with scaling functions $\phi$ and $\widetilde{\phi}$, respectively are said to be biorthogonal to each other if $\left\{\phi(\cdot \ominus \sigma): \sigma \in \Lambda^{+}\right\}$and $\left\{\widetilde{\phi}(\cdot \ominus \sigma): \sigma \in \Lambda^{+}\right\}$are biorthogonal.

Definition 3.6 Let $\phi$ and $\widetilde{\phi}$ be scaling functions for biorthogonal NUMRA's. For each $j \in \mathbb{Z}$, define the operators $P_{j}$ and $\widetilde{P}_{j}$ on $L^{2}\left(\mathbb{R}^{+}\right)$by 


$$
P_{j} f=\sum_{\sigma \in \Lambda^{+}}\left\langle f, \widetilde{\phi}_{j, \sigma}\right\rangle \phi_{j, \sigma} \quad \text { and } \quad \widetilde{P}_{j} f=\sum_{\sigma \in \Lambda^{+}}\left\langle f, \phi_{j, \sigma}\right\rangle \widetilde{\phi}_{j, \sigma}
$$

respectively. It is easy to verify that these operators are uniformly bounded on $L^{2}\left(\mathbb{R}^{+}\right)$and both the series are convergent in $L^{2}\left(\mathbb{R}^{+}\right)$.

\section{Remark 3.7}

The operators $P_{j}$ and $\widetilde{P}_{j}$ satisfy the following properties.

(a) $\quad P_{j} f=f \Longleftrightarrow f \in V_{j}$ and $\widetilde{P}_{j} f=f \Longleftrightarrow f \in \widetilde{V}_{j}$.

(b) $\lim _{j \rightarrow \infty}\left\|P_{j} f-f\right\|_{2}=0$ and $\lim _{j \rightarrow-\infty}\left\|P_{j} f\right\|_{2}=0$ for every $f \in L^{2}\left(\mathbb{R}^{+}\right)$.

Lemma 3.8 Let $\phi$ and $\widetilde{\phi}$ be the scaling functions for biorthogonal NUMRA's and $\psi_{\ell}$ and $\widetilde{\psi}_{\ell}, 1 \leq \ell \leq N-1$ be the associated wavelets satisfying (3.6). Then, we have the following

(a) $\left\{\psi_{\ell, 0, \sigma}: \sigma \in \Lambda^{+}\right\}$is biorthogonal to $\left\{\widetilde{\psi}_{\ell, 0, \gamma}: \gamma \in \Lambda^{+}\right\}$,

(b) $\left\langle\psi_{\ell, 0, \sigma}, \widetilde{\phi}_{0, \gamma}\right\rangle=\left\langle\widetilde{\psi}_{\ell, 0, \sigma}, \phi_{0, \gamma}\right\rangle$, for all $\sigma, \gamma \in \Lambda^{+}$.

Proof we have

$$
\begin{aligned}
& \sum_{t \in \mathbb{Z}} \widehat{\psi}_{\ell}\left(\zeta \oplus \frac{t}{2}\right) \overline{\widehat{\widetilde{\psi}}_{\ell}\left(\zeta \oplus \frac{t}{2}\right)} \\
& =\sum_{t \in \mathbb{Z}}\left\{m_{\ell}\left(\frac{\zeta}{N} \oplus \frac{t}{2 N}\right) \hat{\phi}\left(\frac{\zeta}{N} \oplus \frac{t}{2 N}\right) \overline{\widetilde{m}_{\ell}\left(\frac{\zeta}{N} \oplus \frac{t}{2 N}\right)} \overline{\widehat{\tilde{\phi}}\left(\frac{\zeta}{N} \oplus \frac{t}{2 N}\right)}\right\} \\
& =\sum_{s=0}^{N-1} \sum_{t \in \mathbb{Z}}\left\{m_{\ell}\left(\frac{\zeta}{N} \oplus \frac{t}{2} \oplus \frac{s}{2 N}\right) \hat{\phi}\left(\frac{\zeta}{N} \oplus \frac{t}{2} \oplus \frac{s}{2 N}\right) \overline{\widetilde{m}_{\ell}\left(\frac{\zeta}{N} \oplus \frac{t}{2} \oplus \frac{s}{2 N}\right)} \overline{\widetilde{\tilde{\phi}}\left(\frac{\zeta}{N} \oplus \frac{t}{2} \oplus \frac{s}{2 N}\right)}\right\} \\
& =\sum_{s=0}^{N-1}\left\{m_{\ell}\left(\frac{\zeta}{N} \oplus \frac{s}{2 N}\right) \overline{\widetilde{m}_{\ell}\left(\frac{\zeta}{N} \oplus \frac{s}{2 N}\right)}\right\} \\
& =1 \text {. }
\end{aligned}
$$

Hence, by Lemma 3.1, $\left\{\psi_{\ell, 0, \sigma}: \sigma \in \Lambda^{+}\right\}$is biorthogonal to $\left\{\widetilde{\psi}_{\ell, 0, \sigma}: \sigma \in \Lambda^{+}\right\}$. This proves part (a). To prove part (b), we have for, $\sigma, \gamma \in \Lambda^{+}$ 


$$
\begin{aligned}
& \left\langle\psi_{\ell, 0, \sigma}, \widetilde{\phi}_{0, \gamma}\right\rangle=\left\langle\psi_{\ell}(x \ominus \sigma), \widetilde{\phi}(x \ominus \gamma)\right\rangle \\
& =\left\langle\widehat{\psi}_{\ell} \overline{\chi(\sigma . \zeta)}, \widehat{\widetilde{\phi}} \overline{\chi(\gamma, \zeta)}\right\rangle \\
& =\int_{\mathbb{R}^{+}} m_{\ell}\left(\frac{\zeta}{N}\right) \widehat{\phi}\left(\frac{\zeta}{N}\right) \overline{\chi(\sigma, \zeta)} \overline{\tilde{m}_{0}\left(\frac{\zeta}{N}\right)} \overline{\widehat{\widetilde{\phi}}\left(\frac{\zeta}{N}\right)} \overline{\chi(\gamma, \zeta)} \mathrm{d} \zeta \\
& =\int_{0}^{1 / 2} \sum_{p \in \mathbb{Z}}\left\{m_{\ell}\left(\frac{\zeta}{N} \oplus \frac{p}{2 N}\right) \widehat{\phi}\left(\frac{\zeta}{N} \oplus \frac{p}{2 N}\right)\right. \\
& \left.\overline{\times \widetilde{m}_{0}\left(\frac{\zeta}{N} \oplus \frac{p}{2 N}\right)} \overline{\widehat{\widetilde{\phi}}\left(\frac{\zeta}{N} \oplus \frac{p}{2 N}\right)}\right\} \chi(\gamma \ominus \sigma, \zeta) \mathrm{d} \zeta \\
& =\int_{0}^{1 / 2} \sum_{s=0}^{N-1} \sum_{p \in \mathbb{Z}}\left\{m_{\ell}\left(\frac{\zeta}{N} \oplus \frac{p}{2} \oplus \frac{s}{2 N}\right) \widehat{\phi}\left(\frac{\zeta}{N} \oplus \frac{p}{2} \oplus \frac{s}{2 N}\right)\right. \\
& \left.\times \overline{\widetilde{m}_{0}\left(\frac{\zeta}{N} \oplus \frac{p}{2} \oplus \frac{s}{2 N}\right)} \overline{\widehat{\widetilde{\phi}}\left(\frac{\zeta}{N} \oplus \frac{p}{2} \oplus \frac{s}{2 N}\right)}\right\} \chi(\gamma \ominus \sigma, \zeta) \mathrm{d} \zeta \\
& =\int_{0}^{1 / 2} \sum_{s=0}^{N-1}\left\{m_{\ell}\left(\frac{\zeta}{N} \oplus \frac{s}{2 N}\right) \overline{\tilde{m}_{0}\left(\frac{\zeta}{N} \oplus \frac{s}{2 N}\right)}\right\} \chi(\gamma \ominus \sigma, \zeta) \mathrm{d} \zeta \\
& =0 \text {. }
\end{aligned}
$$

The dual one can also be shown equal to zero in a similar manner. This proves part (b) and hence the proof is completed.

Theorem 3.9 Let $\phi, \widetilde{\phi}, \psi_{\ell}$ and $\widetilde{\psi}_{\ell}, 1 \leq \ell \leq N-1$ be as in Theorem 3.8. Let $\psi_{0}=\phi$ and $\widetilde{\psi}_{0}=\widetilde{\phi}$. Then, for every $f \in L^{2}(\mathbb{R})$, we have

$$
P_{1} f=P_{0} f+\sum_{\ell=1}^{N-1} \sum_{\sigma \in \Lambda^{+}}\left\langle f, \widetilde{\psi}_{\ell, 0, \sigma}\right\rangle \psi_{\ell, 0, \sigma}
$$

and

$$
\widetilde{P}_{1} f=\widetilde{P}_{0} f+\sum_{\ell=1}^{N-1} \sum_{\sigma \in \Lambda^{+}}\left\langle f, \psi_{\ell, 0, \sigma}\right\rangle \widetilde{\psi}_{\ell, 0, \sigma} .
$$

where the series in (3.7) and (3.8) converges in $L^{2}\left(\mathbb{R}^{+}\right)$.

Proof For the sake of convenience, we will only prove (3.7), as (3.8) is an easy consequence. In particular, we will prove it in the weak sense only. For this, let $f, g \in L^{2}(\mathbb{R})$. Then, we have 


$$
\begin{aligned}
& \sum_{\ell=0}^{N-1} \sum_{\sigma \in \Lambda^{+}}\left\langle f, \widetilde{\psi}_{\ell, 0, \sigma}\right\rangle \overline{\left\langle g, \psi_{\ell, 0, \sigma}\right\rangle} \\
& =\sum_{\ell=0}^{N-1} \sum_{\sigma \in \Lambda^{+}}\left\{\int_{\mathbb{R}^{+}} \widehat{f}(\zeta) \overline{\widetilde{\widetilde{\psi}}_{\ell}(\zeta) \chi}(\sigma, \zeta) \mathrm{d} \zeta\right\}\left\{\int_{\mathbb{R}^{+}} \overline{\widehat{g}(\zeta)} \widehat{\psi}_{\ell}(\zeta) \overline{\chi(\sigma, \zeta)} \mathrm{d} \zeta\right\} \\
& =\sum_{\ell=0}^{N-1} \sum_{\sigma \in \Lambda^{+}}\left\{\int_{0}^{1 / 2} \sum_{p \in \mathbb{Z}} \widehat{f}\left(\zeta \oplus \frac{p}{2}\right) \overline{\hat{\tilde{\psi}}_{\ell}\left(\zeta \oplus \frac{p}{2}\right)} \chi(\sigma, \zeta) \mathrm{d} \zeta\right\} \\
& \times\left\{\int_{0}^{1 / 2} \sum_{q \in \mathbb{Z}} \bar{g}\left(\zeta \oplus \frac{q}{2}\right) \widehat{\psi}_{\ell}\left(\zeta \oplus \frac{q}{2}\right) \overline{\chi(\sigma, \zeta)} \mathrm{d} \zeta\right\} \\
& =\sum_{\ell=0}^{N-1} \int_{0}^{1 / 2}\left\{\sum_{p \in \mathbb{Z}} \widehat{f}\left(\zeta \oplus \frac{p}{2}\right) \overline{\overline{\widetilde{\psi}}_{\ell}\left(\zeta \oplus \frac{p}{2}\right)}\right\}\left\{\sum_{q \in \mathbb{Z}} \overline{\widehat{g}\left(\zeta \oplus \frac{q}{2}\right)} \widehat{\psi}_{\ell}\left(\zeta \oplus \frac{q}{2}\right)\right\} \mathrm{d} \zeta \\
& =\int_{0}^{1 / 2} \sum_{\ell=0}^{N-1}\left\{\sum_{p \in \mathbb{Z}} \widehat{f}\left(\zeta \oplus \frac{p}{2}\right) \overline{\widetilde{m}_{\ell}\left(\frac{\zeta}{N} \oplus \frac{p}{2 N}\right)} \overline{\widetilde{\widehat{\phi}}\left(\frac{\zeta}{N} \oplus \frac{p}{2 N}\right)}\right. \\
& \left.\times \sum_{q \in \mathbb{Z}} \overline{\widehat{g}\left(\zeta \oplus \frac{q}{2}\right)} m_{\ell}\left(\frac{\zeta}{N} \oplus \frac{q}{2 N}\right) \widehat{\phi}\left(\frac{\zeta}{N} \oplus \frac{q}{2 N}\right)\right\} \mathrm{d} \zeta \\
& =\int_{0}^{1 / 2} \sum_{\ell=0}^{N-1}\left\{\sum_{r=0}^{N-1} \sum_{p^{\prime} \in \mathbb{Z}} \widehat{f}\left(\zeta \oplus \frac{p^{\prime}}{2} N \oplus \frac{r}{2}\right) \overline{\widetilde{m}_{\ell}\left(\frac{\zeta}{N} \oplus \frac{r}{2 N} \oplus \frac{p^{\prime}}{2}\right)} \overline{\widetilde{\phi}\left(\frac{\zeta}{N} \oplus \frac{r}{2 N} \oplus \frac{p^{\prime}}{2}\right)}\right. \\
& \left.\times \sum_{s=0}^{N-1} \sum_{q^{\prime} \in \mathbb{N}_{0}} \bar{g}\left(\zeta \oplus \frac{q^{\prime}}{2} N \oplus \frac{s}{2}\right) m_{\ell}\left(\frac{\zeta}{N} \oplus \frac{s}{2 N} \oplus \frac{q^{\prime}}{2}\right) \hat{\phi}\left(\frac{\zeta}{N} \oplus \frac{s}{2 N} \oplus \frac{q^{\prime}}{2}\right)\right\} \mathrm{d} \zeta \\
& =\int_{0}^{1 / 2} \sum_{r=0}^{N-1} \sum_{p^{\prime} \in \mathbb{N}_{0}} \sum_{s=0}^{N-1} \sum_{q^{\prime} \in \mathbb{N}_{0}}\left\{\sum_{\ell=0}^{N-1} \overline{\tilde{m}_{\ell}\left(\frac{\zeta}{N} \oplus \frac{r}{2 N}\right)} m_{\ell}\left(\frac{\zeta}{N} \oplus \frac{s}{2 N}\right)\right\}
\end{aligned}
$$

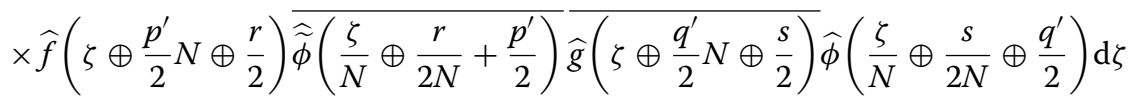

$$
\begin{aligned}
& =\int_{0}^{1 / 2} \sum_{p^{\prime} \in \mathbb{N}_{0}} \sum_{q^{\prime} \in \mathbb{N}_{0}} \sum_{s=0}^{N-1} \widehat{f}\left(\zeta \oplus \frac{p^{\prime}}{2} N \oplus \frac{s}{2}\right) \overline{\widehat{\widetilde{\phi}}\left(\frac{\zeta}{N} \oplus \frac{s}{2 N} \oplus \frac{p^{\prime}}{2}\right)} \\
& \times \overline{\widehat{g}\left(\zeta \oplus \frac{q^{\prime}}{2} N \oplus \frac{s}{2}\right)} \widehat{\phi}\left(\frac{\zeta}{N} \oplus \frac{s}{2 N} \oplus \frac{p^{\prime}}{2}\right) \mathrm{d} \zeta \\
& =\sum_{s=0}^{N-1} \int_{0}^{s+1 / 2} \sum_{p^{\prime} \in \mathbb{N}_{0}} \sum_{q^{\prime} \in \mathbb{N}_{0}} \widehat{f}\left(\zeta \oplus \frac{p^{\prime}}{2} N\right) \overline{\widetilde{\widetilde{\phi}}\left(\frac{\zeta}{N} \oplus \frac{p^{\prime}}{2}\right)} \widehat{\widehat{g}\left(\zeta \oplus \frac{q^{\prime}}{2} N\right)} \widehat{\phi}\left(\frac{\zeta}{N} \oplus \frac{p^{\prime}}{2}\right) \mathrm{d} \zeta .
\end{aligned}
$$


Furthermore, we have

$$
\begin{aligned}
& \sum_{\sigma \in \Lambda^{+}}\left\langle f, \widetilde{\phi}_{1, \sigma}\right\rangle \overline{\left\langle g, \phi_{1, \sigma}\right\rangle} \\
& =\sum_{\sigma \in \Lambda^{+}}\left\{\int_{\mathbb{R}} \widehat{f}(\zeta) \overline{\widetilde{\phi}}\left(\frac{\zeta}{N}\right) e^{2 \pi i \zeta / N} \mathrm{~d} \zeta\right\}\left\{\int_{\mathbb{R}} \overline{\widehat{g}(\zeta)} \widehat{\phi}\left(\frac{\zeta}{N}\right) e^{-2 \pi i \zeta / N} \mathrm{~d} \zeta\right\}
\end{aligned}
$$

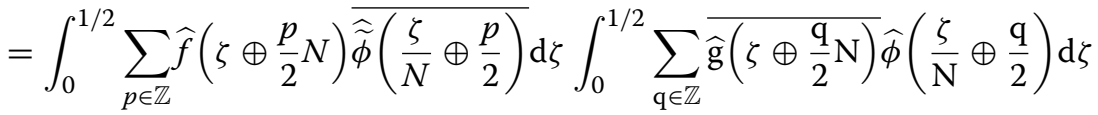

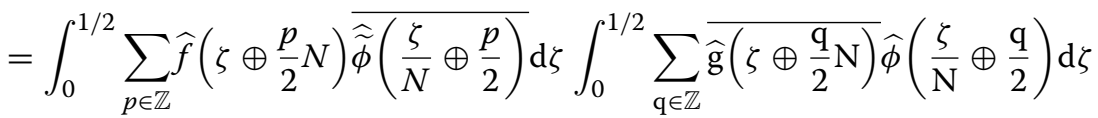

$$
\begin{aligned}
& =\int_{0}^{1 / 2} \sum_{p \in \mathbb{Z}} \sum_{q \in \mathbb{Z}} \widehat{f}\left(\zeta \oplus \frac{p}{2} N\right) \overline{\widehat{\widetilde{\phi}}\left(\frac{\zeta}{N} \oplus \frac{p}{2}\right)} \overline{\widehat{g}\left(\zeta \oplus \frac{q}{2} N\right)} \widehat{\phi}\left(\frac{\zeta}{N} \oplus \frac{q}{2}\right) \mathrm{d} \zeta .
\end{aligned}
$$

Combing (3.9) and (3.10), we get the desired result.

Theorem 3.10 Let $\phi$ and $\widetilde{\phi}$ be the scaling functions for biorthogonal NUMRA's and $\psi_{\ell}$ and $\widetilde{\psi}_{\ell}, 1 \leq \ell \leq N-1$ be the associated wavelets satisfying the matrix condition (3.6). Then, the collection $\left\{\psi_{\ell, j, \sigma}: 1 \leq \ell \leq N-1, j \in \mathbb{Z}, \sigma \in \Lambda^{+}\right\}$and $\left\{\widetilde{\psi}_{\ell, j, \sigma}: 1 \leq \ell \leq N-1, j \in \mathbb{Z}, \sigma \in \Lambda^{+}\right\}$are biorthogonal. Moreover, if

$$
|\widehat{\phi}(\zeta)| \leq K(1+|\zeta|)^{-\frac{1}{2}-\epsilon},|\widehat{\widetilde{\phi}}(\zeta)| \leq K(1+|\zeta|)^{-\frac{1}{2}-\epsilon},\left|\widehat{\psi}_{\ell}(\zeta)\right| \leq K|\zeta| \text { and }|\widehat{\widetilde{\psi}}(\zeta)| \leq K|\zeta|,
$$

for some constant $K>0, \epsilon>0$ and for a.e. $\zeta \in \mathbb{R}$, then $\left\{\psi_{\ell, j, \sigma}: 1 \leq \ell \leq N-1, j \in \mathbb{Z}, \sigma \in \Lambda^{+}\right\} \quad$ and $\quad\left\{\widetilde{\psi}_{\ell, j, \sigma}: 1 \leq \ell \leq N-1, j \in \mathbb{Z}, \sigma \in \Lambda^{+}\right\}$ form Riesz bases for $L^{2}(\mathbb{R})$.

Proof First we show that $\left\{\psi_{\ell, j, \sigma}: 1 \leq \ell \leq N-1, j \in \mathbb{Z}, \sigma \in \Lambda^{+}\right\}$and $\left\{\widetilde{\psi}_{\ell, j, \sigma}: 1 \leq \ell \leq N-1, j \in \mathbb{Z}, \sigma \in \Lambda^{+}\right\}$are biorthogonal to each other. For this, we will show that for each $\ell, 1 \leq \ell \leq N-1$ and $j \in \mathbb{Z}$,

$$
\left\langle\psi_{\ell, j, \sigma}, \widetilde{\psi}_{\ell, j, \gamma}\right\rangle=\delta_{\sigma, \gamma}
$$

In fact, we have already proved (3.12) for $j=0$. For $j \neq 0$, we have

$$
\left\langle\psi_{\ell, j, \sigma}, \widetilde{\psi}_{\ell, j, \gamma}\right\rangle=\left\langle D_{-j} \psi_{\ell, 0, \sigma}, D_{-j} \widetilde{\psi}_{\ell, 0, \gamma}\right\rangle=\left\langle\psi_{\ell, 0, \sigma}, \widetilde{\psi}_{\ell, 0, \gamma}\right\rangle=\delta_{\sigma, \gamma}
$$

Also, for fixed $\sigma, \gamma \in \Lambda^{+}$and $j, j^{\prime} \in \mathbb{Z}$ with $j<j^{\prime}$, we claim that

$$
\left\langle\psi_{\ell, j, \sigma}, \widetilde{\psi}_{\ell^{\prime}, j^{\prime}, \gamma}\right\rangle=0 \text {. }
$$

As $\psi_{\ell, 0, \sigma} \in \mathcal{V}_{1}$, hence $\psi_{\ell, j, \sigma}=D_{-j} \psi_{\ell, 0, \sigma} \in \mathcal{V}_{j+1} \subseteq \mathcal{V}_{j^{\prime}}$. Therefore, it is enough to show that $\widetilde{\psi}_{\ell^{\prime}, j^{\prime}, \gamma}$ is orthogonal to every element of $\mathcal{V}_{j^{\prime}}$. Let $g \in \mathcal{V}_{j^{\prime}}$. Since $\left\{\phi_{j^{\prime}, \sigma}: \sigma \in \Lambda^{+}\right\}$ is a Riesz basis for $\mathcal{V}_{j^{\prime}}$, hence there exists an $l^{2}$-sequence $\left\{d_{\sigma}: \sigma \in \Lambda^{+}\right\}$such that $g=\sum_{\sigma \in \Lambda^{+}} d_{\sigma} \phi_{j^{\prime}, \sigma}$ in $L^{2}\left(\mathbb{R}^{+}\right)$. Using part (b) of Lemma 3.6, we have 


$$
\left\langle\widetilde{\psi}_{\ell^{\prime}, j^{\prime}, \gamma}, \phi_{j^{\prime}, \sigma}\right\rangle=\left\langle D_{-j^{\prime}} \widetilde{\psi}_{\ell^{\prime}, 0, \gamma}, D_{-j^{\prime}} \phi_{0, \sigma}\right\rangle=0 .
$$

Therefore,

$$
\left\langle\widetilde{\psi}_{\ell^{\prime}, j^{\prime}, \gamma}, g\right\rangle=\left\langle\widetilde{\psi}_{\ell^{\prime}, j^{\prime}, \gamma}, \sum_{\sigma \in \Lambda^{+}} d_{\sigma} \phi_{j^{\prime}, \sigma}\right\rangle=\sum_{\sigma \in \Lambda^{+}} d_{\sigma}\left\langle\widetilde{\psi}_{\ell^{\prime}, j^{\prime}, \gamma}, \phi_{j^{\prime}, \sigma}\right\rangle=0 .
$$

We now show that these two collections form Riesz bases for $L^{2}\left(\mathbb{R}^{+}\right)$. The linear independence is clear from the fact that these collections are biorthogonal to each other. So, we have to check the frame inequalities only i.e., there exists constants $C, \widetilde{C}, D, \widetilde{D}>0$ such that

$$
C\|g\|_{2}^{2} \leq \sum_{\ell=1}^{N-1} \sum_{j \in \mathbb{Z}} \sum_{\sigma \in \Lambda^{+}}\left|\left\langle g, \psi_{\ell, j, \sigma}\right\rangle\right|^{2} \leq D\|g\|_{2}^{2}, \quad \forall f \in L^{2}\left(\mathbb{R}^{+}\right),
$$

and

$$
\widetilde{C}\|g\|_{2}^{2} \leq \sum_{\ell=1}^{N-1} \sum_{j \in \mathbb{Z}} \sum_{\sigma \in \Lambda^{+}}\left|\left\langle g, \widetilde{\psi}_{\ell, j, \sigma}\right\rangle\right|^{2} \leq \widetilde{D}\|g\|_{2}^{2}, \quad \forall f \in L^{2}\left(\mathbb{R}^{+}\right) .
$$

Let us first check the existence of the upper bounds in (3.14) and (3.15). For this, we have

$$
\begin{aligned}
\sum_{\sigma \in \Lambda^{+}}\left|\left\langle g, \widetilde{\psi}_{\ell, j, \sigma}\right\rangle\right|^{2} & =\sum_{\sigma \in \Lambda^{+}}\left|\int_{\mathbb{R}^{+}} \widehat{g}(\zeta)(N)^{-j / 2} \overline{\widehat{\psi}_{\ell}\left(N^{-j} \zeta\right)} \chi\left(\sigma, N^{-j} \zeta\right) \mathrm{d} \zeta\right|^{2} \\
& =N^{-j} \sum_{\sigma \in \Lambda^{+}}\left|\int_{0}^{1 / 2} \sum_{p \in \mathbb{Z}} \widehat{g}\left(\zeta \oplus(N)^{j} \frac{p}{2}\right) \overline{\widehat{\psi}_{\ell}\left((N)^{-j} \zeta \oplus \frac{p}{2}\right)} \chi\left(\sigma, N^{-j} \zeta\right) \mathrm{d} \zeta\right|^{2} \\
& =\int_{0}^{1 / 2}\left|\sum_{p \in \mathbb{Z}} \widehat{g}\left(\zeta \oplus(N)^{j} \frac{p}{2}\right) \overline{\widehat{\psi}_{\ell}\left((N)^{-j} \zeta \oplus \frac{p}{2}\right)}\right|^{2} \mathrm{~d} \zeta \\
& =\int_{0}^{1 / 2}\left\{\sum_{p \in \mathbb{Z}}\left|\widehat{g}\left(\zeta \oplus(N)^{j} \frac{p}{2}\right)\right|^{2}\left|\widehat{\psi}_{\ell}\left((N)^{-j} \zeta \oplus \frac{p}{2}\right)\right|^{2 \delta}\right\} \\
& \times\left\{\sum_{q \in \mathbb{Z}}\left|\widehat{\psi}_{\ell}\left((N)^{-j} \zeta \oplus \frac{q}{2}\right)\right|^{2(1-\delta)}\right\} \mathrm{d} \zeta \\
= & \int_{\mathbb{R}^{+}}|\widehat{g}(\zeta)|^{2}\left|\widehat{\psi}_{\ell}\left((N)^{-j} \zeta\right)\right|^{2 \delta} \sum_{q \in \mathbb{Z}}\left|\widehat{\psi}_{\ell}\left((N)^{-j} \zeta \oplus \frac{q}{2}\right)\right|^{2(1-\delta)} \mathrm{d} \zeta .
\end{aligned}
$$

By our assumption (3.11), we have $\left|\widehat{\psi}_{\ell}(\zeta)\right| \leq K\left(1+\left|(N)^{-1} \zeta\right|\right)^{-1 / 2-\epsilon}$ and therefore, it follows that $\sum_{q \in \mathbb{Z}}\left|\widehat{\psi}_{\ell}\left((N)^{-j} \zeta+q / 2\right)\right|^{2(1-\delta)}$ is uniformly bounded if $\delta<2 \epsilon(1+2 \epsilon)^{-1}$. Thus, there exists a constant $K>0$ such that 


$$
\begin{aligned}
\sum_{\sigma \in \Lambda^{+}}\left|\left\langle g, \widetilde{\psi}_{\ell, j, \sigma}\right\rangle\right|^{2} & \leq K \int_{\mathbb{R}^{+}}|\widehat{g}(\zeta)|^{2} \sum_{j \in \mathbb{Z}}\left|\widehat{\psi}_{\ell}\left((N)^{-j} \zeta\right)\right|^{2 \delta} \mathrm{d} \zeta \\
& \leq K \sup \left\{\sum_{j \in \mathbb{Z}}\left|\widehat{\psi}_{\ell}\left((N)^{-j} \zeta\right)\right|^{2 \delta}: 1 \leq \zeta \leq N\right\}\|g\|_{2}^{2} .
\end{aligned}
$$

Also for $\zeta \in[1, N]$, we have

$$
\begin{aligned}
\sum_{j=-\infty}^{0}\left|\widehat{\psi}_{\ell}\left((N)^{-j} \zeta\right)\right|^{2 \delta} & \leq \sum_{j=-\infty}^{0} \frac{K^{2 \delta}}{\left(1+\left|(N)^{j-1} \zeta\right|\right)^{\delta(1+2 \epsilon)}} \\
& \leq \sum_{j=-\infty}^{0} \frac{K^{2 \delta}}{(N)^{(j-1) \delta(1+2 \epsilon)}} \\
& \leq K^{2 \delta} \frac{q^{\delta(1+2 \epsilon)}}{1-(N)^{-\delta(1+2 \epsilon)}}
\end{aligned}
$$

Furthermore, we have

$$
\sum_{j=1}^{\infty}\left|\widehat{\psi}_{\ell}\left((N)^{-j} \zeta\right)\right|^{2 \delta} \leq \sum_{j=1}^{\infty}\left(K(N)^{-j}|\zeta|\right)^{2 \delta} \leq K^{2 \delta} \sum_{j=1}^{\infty}(N)^{(-j+1) 2 \delta}=K^{2 \delta} \frac{1}{1-(N)^{-2 \delta}}
$$

and hence, it follows that $\sup \left\{\sum_{j \in \mathbb{Z}}\left|\widehat{\psi}_{\ell}\left((N)^{-j} \zeta\right)\right|^{2 \delta}: 1 \leq \zeta \leq N\right\}$ is finite. Therefore, there exist $D>0$ such that of (3.15) holds. Similarly, we can show for dual one also. The existence of lower bounds for both the cases can be shown in similar fashion. Thus, we have

$$
\begin{aligned}
\|g\|_{2}^{2} & =\langle g, g\rangle \\
& =\left\langle\sum_{\ell=1}^{N-1} \sum_{j \in \mathbb{Z}} \sum_{\sigma \in \Lambda^{+}}\left\langle g, \widetilde{\psi}_{\ell, j, \sigma}\right\rangle \psi_{\ell, j, \sigma}, g\right\rangle \\
& =\sum_{\ell=1}^{N-1} \sum_{j \in \mathbb{Z}} \sum_{\sigma \in \Lambda^{+}}\left\langle g, \widetilde{\psi}_{\ell, j, \sigma}\right\rangle\left\langle\psi_{\ell, j, \sigma}, g\right\rangle \\
& \leq\left(\sum_{\ell=1}^{N-1} \sum_{j \in \mathbb{Z}} \sum_{\sigma \in \Lambda^{+}}\left|\left\langle g, \widetilde{\psi}_{\ell, j, \sigma}\right\rangle\right|^{2}\right)^{1 / 2}\left(\sum_{\ell=1}^{N-1} \sum_{j \in \mathbb{Z}} \sum_{\sigma \in \Lambda^{+}}\left|\left\langle g, \psi_{\ell, j, \sigma}\right\rangle\right|^{2}\right)^{1 / 2} \\
& \leq(\widetilde{D})^{1 / 2}\|g\|_{2}\left(\sum_{\ell=1}^{N-1} \sum_{j \in \mathbb{Z}} \sum_{\sigma \in \Lambda^{+}}\left|\left\langle g, \psi_{\ell, j, \sigma}\right\rangle\right|^{2}\right)^{1 / 2}
\end{aligned}
$$

Hence,

$$
\frac{1}{\widetilde{D}}\|g\|_{2}^{2} \leq \sum_{\ell=1}^{N-1} \sum_{j \in \mathbb{Z}} \sum_{\sigma \in \Lambda^{+}}\left|\left\langle g, \psi_{\ell, j, \sigma}\right\rangle\right|^{2} .
$$

The dual case can be proved in similar lines. This completes the proof. 


\section{Conclusions}

In this paper, we develop the comprehensive theory of nonuniform biorthogonal wavelets on the positive half line. We provide the complete characterization for the translates of a single function to form Reisz basis and the associated biorthogonal families with respect to NUMRA in $L^{2}\left(\mathbb{R}^{+}\right)$. Under some mild assuptions on wavelets associated with NUMRA and the scaling function, we show the wavelets can generate Reisz bases.The results established in this paper are theoretical in nature and will definitely provide new directions to the development of wavelet analysis and widen its field of applications.

\section{Abbreviations}

OA: Owais Ahmad; NAS: Neyaz Ahmad Sheikh; MA: Mobin Ahmad.

\section{Acknowledgements}

Not Applicable.

\section{Author's contributions}

OA wrote abstract and mathematical analysis of the results. NAS wrote the introduction and fixed many grammatical errors. MA wrote conclusion and typed the paper in Latex. All authors read and approved the final manuscript.

Funding

There are no funding sources for this manuscript.

Availability of data and materials

Data sharing is not applicable to this article as no datasets were generated or analyzed during the current study.

\section{Declarations}

Competing interests

The author declares that there are no competing interests.

\section{Author details}

'Department of Mathematics, National Institute of Technology, Srinagar, Jammu and Kashmir 190006, India. ${ }^{2}$ Department of Mathematics, Faculty of Science, Jazan University, Jazan 45142, Saudi Arabia.

Received: 6 October 2020 Accepted: 28 September 2021

Published online: 15 October 2021

\section{References}

1. Bownik, M., Garrigos, G.: Biorthogonal wavelets, MRA's and shift-invariant spaces. Studia Math. 160, 231-248 (2004)

2. Cohen, A., Daubechies, I., Feauveau, J.C.: Biorthogonal bases of compactly supported wavelets. Commun. Pure Appl. Math. 45, 485-560 (1992)

3. Chui, C.K., Wang, J.Z.: On compactly supported spline wavelets and a duality principle. Trans. Amer. Math. Soc. 330(2), 903-915 (1992)

4. Karoui, A., Vaillancourt, R.: Families of Biorthogonal Wavelets. Comput. Math. Applic. 28(4), 25-39 (1994)

5. Gabardo, J.P., Nashed, M.: Nonuniform multiresolution analyses and spectral pairs. J. Funct. Anal. 158, 209-241 (1998)

6. Shah, F., Abdullah, A.: Nonuniform multiresolution analysis on local fields of positive characteristic. Complex Anal. Opert. Theory. 9, 1589-1608 (2015)

7. Farkov, Y.A., Maksimov, A.Y., Stroganov, S.A.: On biorthogonal wavelets related to the Walsh functions. Int. J. Wavelets Multiresolut. Inf. Process. 9(3), 485-499 (2011)

8. Meenakshi, Manchanda, P., Siddiqi, A.H.: Wavelets associated with nonuniform multiresolution analysis on positive half-line. Int. J. Wavelets Multiresolut. Inf. Process. 10(2), 1250018 (2012)

9. Shah, F.A., Ahmad, O., Sheikh, N.A.: Some new inequalities for wavelet frames on local fields. Anal. Theory Appl. 33(2), 134-148 (2017)

10. Ahmad, O., Sheikh, N.A., Ali, M.A.: Nonuniform nonhomogeneous dual wavelet frames in Sobolev spaces in $L^{2}(\mathbb{K})$. Afrika Math. (2020). https://doi.org/10.1007/s13370-020-00786-1

11. Ahmad, O., Sheikh, N.A.: On Characterization of nonuniform tight wavelet frames on local fields. Anal. Theory Appl. 34, 135-146 (2018)

12. Ahmad, O., Ahmad, N.: Construction of nonuniform wavelet frames on non-archimedean fields. Math. Phy. Anal. Geometry 23(47) (2020)

13. Ahmad, O., Sheikh, N.A., Nisar, K.S., Shah, F.A.: Biorthogonal wavelets on spectrum. Math. Methods Appl. Sci. https://doi.org/10.1002/mma.7046 (2021) 
14. Ahmad, O.: Nonuniform periodic wavelet frames on non-archimedean fields. Annales Universitatis Mariae CurieSklodowska, sectio A - Mathematica. https://doi.org/10.17951/a.2020.74.2.1-17(2020)

15. Ahmad, O., Ahmad, N.: Nonuniform p-tight wavelet frames on positive half line. TWMS J. Appl. Eng. Math. (To appear)

16. Ahmad, O., Ahmad, N.: Explicit construction of tight nonuniform framelet packets on local fields. Oper. Matric. 15(1), 131-149 (2021)

17. Ahmad, O., Bhat, M.Y., Sheikh, N.A.: Construction of Parseval Framelets Associated with GMRA on local fields of positive characteristic. Numer. Funct. Anal. Optim. (2021). https://doi.org/10.1080/01630563.2021.1878370

18. Shah, F.A., Ahmad, O.: Wave packet systems on local fields. J. Geom. Phys. 120, 5-18 (2017)

19. Shah, F.A., Ahmad, O., Jorgenson, P.E.: Fractional wave packet frames in $L^{2}(\mathbb{R})$. J. Math. Phys. 59, 073509 (2018). https://doi.org/10.1063/1.5047649

20. Shah, F.A., Ahmad, O., Rahimi, A.: Frames associated with shift invariant spaces on local fields. Filomat 32(9), 3097-3110 (2018)

21. Ahmad, O., Shah, F.A., Sheikh, N.A.: Gabor frames on non-Archimedean fields. Int. J. Geom. Methods Mod. Phys. 15, $1850079(2018)$

22. Agarwal, P., Deniz, S., Jain, S., Alderremy, A.A.: Aly Shaban, A new analysis of a partial differential equation arising in biology and population genetics via semi analytical techniques. Physica A: Stat. Mech. Appl. 542, 122769 (2020)

23. Saad, K.M., Iyiola, O.S., Agarwal, P.: An effective homotopy analysis method to solve the cubic isothermal autocatalytic chemical system. AIMS Math. 3(1), 183-194 (2018)

24. Chu, Y.M., Shah, N.A., Agarwal, P., Chung, J.D.: Analysis of fractional multi-dimensional Navier-Stokes equation. Adv. Differ. Equ. 1, 1-18 (2021)

25. Abd-Elhameed, W.M., Doha, E,H., Youssri Y.H.: New spectral second kind Chebyshev wavelets algorithm for solving linear and nonlinear second-order differential equations involving singular and Bratu type equations. Abst. Appl. Anal. Article ID 715756 (2013)

26. Abd-Elhameed, W.M., Doha, E,H., Youssri Y.H.: New wavelets collocation method for solving second-order multipoint boundary value problems using Chebyshev polynomials of third and fourth kinds. Abst. Appl. Anal. Article ID 542839 (2013)

27. Abd-Elhameed, W.M., Youssri Y.H.: New ultraspherical wavelets spectral solutions for fractional Riccati differential equations. Abst. Appl. Anal. Article ID 626275 (2014)

28. Abd-Elhameed, W.M., Youssri Y.H., Doha, E.H.: Ultraspherical wavelets method for solving lane-emden type equations. Roman. J. Phys. 60(9-10), 1298-1314 (2015)

29. Abd-Elhameed, W.M., Youssri Y.H., Doha, E,H.: Accurate spectral solutions of first-and second-order initial value problems by the ultraspherical wavelets-Gauss collocation method. Appl. Appl. Math. 10(2), 835-851 (2015)

30. Doha, E,H., Abd-Elhameed, W.M., Youssri Y.H.: New ultraspherical wavelets collocation method for solving 2 nthorder initial and boundary value problems. J. Egypt. Math. Soc. 24(2), 319-327 (2016)

31. Golubov, B.I., Efimov, A.V., Skvortsov, V.A.: Walsh Series and Transforms: Theory and Applications. Kluwer, Dordrecht (1991)

32. Schipp, F., Wade, W.R., Simon, P.: Walsh Series: An Introduction to Dyadic Harmonic Analysis. Adam Hilger, Bristol and New York (1990)

33. Restrepo, J.E., Piedrahita, A., Agarwal, P.: Multidimensional Fourier transform and fractional derivative. Proc. Jangjeon Math. Soc. 22(2), 269-279 (2019)

34. Ruzhansky, M., Cho, Y.J., Agarwal, P., Area, I.: Advances in Real and Complex Analysis with Applications

\section{Publisher's Note}

Springer Nature remains neutral with regard to jurisdictional claims in published maps and institutional affiliations.

\section{Submit your manuscript to a SpringerOpen ${ }^{\circ}$ journal and benefit from:}

- Convenient online submission

- Rigorous peer review

- Open access: articles freely available online

- High visibility within the field

Retaining the copyright to your article

Submit your next manuscript at $\boldsymbol{\Delta}$ springeropen.com 